\title{
New combinations for infraspecific taxa in the genus Euphorbia L. (Euphorbiaceae)
}

\author{
ROB C. H. M. OUDEJANS
}

Resum

OudEJANS, R. C. H. M. (1992). Combinacions noves de tàxons infraespecífics en el gènere Euphorbia L. (Euphorbiaceae). Collect. Bot. (Barcelona) 21:183-189.

Es proposen als voltant de 80 combinacions noves de tàxons infraespecífics en el gènere Euphorbia L. com anticipació a la publicació del catàleg mundial de tots el tàxons infraespecífics descrits i usats en la tribu Euphorbieae (Euphorbiaceae). A més s'inclou una nota referent a E. fischeriana Steudel.

Mots claus: Euphorbia, Euphorbiaceae, combinacions noves, tàxons infraespecífics.

\begin{abstract}
Oudejans, R. C. H. M. (1992). New combinations for infraspecific taxa in the genus Euphorbia L. (Euphorbiaceae). Collect. Bot. (Barcelona) 21:183-189.

About 80 new combinations for infraspecific taxa in the genus Euphorbia L. are made in anticipation of publication of a world catalogue of all infraspecific taxa described and used in the tribe Euphorbieae (Euphorbiaceae). Moreover, a note on E. fischeriana Steudel is included.
\end{abstract}

Keywords: Euphorbia, Euphorbiaceae, new combinations, infraspecific taxa.

\section{INTRODUCTION}

Many years of research done for the publication of my 'World catalogue of species names published in the tribe Euphorbieae (Euphorbiaceae) with their geographical distribution' (Oudejans, 1990) have revealed also most of the data necessary for a compilation, now in preparation, of all infraspecific taxa described in that tribe. Several new combinations appeared to be necessary of which a first set is published in this paper, arranged alphabetically by species name.

\section{LIST OF NEW COMBINATIONS}

Euphorbia adenoptera Bertoloni ssp. canescens (Proctor) Oudejans comb. nov. Basionym: Chamaesyce adenoptera (Bertoloni) Small ssp. canescens G. R. Proctor 1983, Moscosoa 2(1): 23-24.

R. C. H. M. OUdEJANS: van Konijnenburglaan 12, 3925 XB Scherpenzeel, The Netherlands. 
Euphorbia amygdaloides Linnaeus f. biennis (Maire) Oudejans stat. nov. Basionym: E. amygdaloides var. biennis R. [C. J. E.] Maire 1935, Bull. Soc. Hist. Nat. Afr. Nord 26: 228.

Euphorbia amygdaloides Linnaeus f. minor (Gaudin) Oudejans comb. \& stat. nov. Basionym: $E$. sylvatica Linnaeus ['Dec.'] var. minor J. F. A. [G. P.] Gaudin 1828, Fl. helv. 3: 281.

Euphorbia amygdaloides Linnaeus f. purpurata (Cosson \& Germain) Oudejans comb. \& stat. nov. Basionym: E. sylvatica Linnaeus subvar. purpurata E. Cosson \& [J. N. E.] Germain 1861, Fl. descr. anal. Paris (ed. 2): 601.

Euphorbia angulata Jacquin $\mathrm{f}$. nemoralis (Kitaibel in Kanitz) Oudejans comb. \& stat. nov. Basionym: E. nemoralis P. Kitaibel in A. Kanitz 1863, Linnaea 32: 557-558.

Euphorbia atlantis Maire var. leiocarpa (Boissier in DC) Oudejans comb. nov. Basionym: E. atlantica Cosson ex Boissier in DC var. leiocarpa [P.] E. Boissier in A. P. de Candolle 1862, Prodr. 15(2): 130.

Euphorbia atlantis Maire var. major (Boissier in DC) Oudejans comb. nov. Basionym: $E$. atlantica Cosson ex Boissier in DC var. major [P.] E. Boissier in A. P. de Candolle 1862, Prodr. 15(2): 130.

Euphorbia brachycera Engelmann in Emory f. dichotoma (Daniels) Oudejans comb. nov. Basionym: Tithymalus philorus Cockerell f. dichotomus ['dichotoma' sic] F. P. Daniels 1911, Univ. Missouri Stud., Sci. Ser. 2(2): 313.

Euphorbia dulcis Linnaeus f. lanuginosa (Petermann) Oudejans stat. nov. Basionym: $E$. dulcis Linnaeus ['Jacq.'] var. lanuginosa W. L. Petermann 1841, Anal. Pfl. Schl. Leipzig: 395.

Euphorbia ebracteolata Hayata var. anhweiensis (Hurusawa) Oudejans comb. nov. Basionym: Galarrhoeus ['Galarhoeus'] ebracteolatus (Hayata) Hara var. anwheiensis I. Hurusawa 1954, J. Fac. Sci. Univ. Tokyo (Sect. 3 Bot.) 6(6): 251.

Euphorbia fischeriana Steudel f. glaberrima (Maximowicz) Oudejans comb. \& stat. nov. Basionym: E. pallasii Turczaninow var. glaberrima C. J. Maximowicz 1883, Mélanges Bot. 11: 833.

Note: I prefer to use the name E. fischeriana Steudel, which was originally proposed by Steudel (1840: 611 ) for the species $E$. verticillata Fischer (1812: 81 ), because it was a later homonym. Steudel clearly referred E. verticillata Fischer as a synonym. However, E. pallasii was proposed earlier by Turczaninow (1838: 100) for E. verticillata Pallas, which according to my information never has been published by Pallas. Although, as Croizat (1940: 492) pointed out, E. verticillata Pallas probably is the same species as E. verticillata Fischer, no proof for this can be found. Several later authors follow Croizat, but if they prefer to do so, another, yet earlier, name E. pallasiana Turczaninow should be used, which was proposed by Fischer \& Meyer (1835: 8) by using the same synonym E. verticillata Pallas.

Euphorbia fischeriana Steudel f. pilosa (Regel) Oudejans comb. \& stat. nov. Basionym: $E$. pallasii Turczaninow var. pilosa E. [A.] von Regel 1861, Mém. Acad. Imp. Sci. St. Petersb. (Ser. 7) 4(4): 128.

Euphorbia gaubae (Soják) A. Radcliffe-Smith var. velutina (Bornmüller \& Gauba) Oudejans comb. nov. Basionym: E. halophila Bornmüller \& Gauba var. velutina J. [F. N.] Bornmüller \& E. Gauba 1939, Feddes Repert. 47: 62.

Euphorbia helioscopia Linnaeus f. litoralis (Hurusawa) Oudejans comb. nov. Basionym: $G a-$ larrhoeus ['Galarhoeus'] helioscopius (Linnaeus) Haworth f. litoralis I. Hurusawa 1954, J. Fac. Sci. Univ. Tokyo (Sect. 3 Bot.) 6(6): 238. 
Euphorbia helioscopia Linnaeus f. major (Mutel) Oudejans stat. nov. Basionym: E. helioscopia var. major [P.] A. [V.] Mutel 1836, Fl. franç. 3: 150.

Euphorbia hieronymi Subils f. parvifolia (Hieronymus) Oudejans comb. nov. Basionym: $E$. dioica Hieronymus f. parvifolia G. [H. E. W.] Hieronymus 1885, Icon. descr. pl. Argent. 1: 47.

Euphorbia hirsuta Nathorst in Linnaeus f. crispata (Willdenow ex Hornemann) Oudejans comb. \& stat. nov. Basionym: E. crispata Willdenow ex J. W. Hornemann 1819, Hort. bot. hafn., Suppl.: 58 .

Euphorbia hirsuta Nathorst in Linnaeus f. glabrescens (Saint-Lager) Oudejans comb. \& stat. nov. Basionym: E. pubescens Vahl ['Desf.'] var. glabrescens [J. B.] Saint-Lager 1883, Cat. fl. bass. Rhône: 665 .

Euphorbia hirsuta Nathorst in Linnaeus f. integrifolia (Deysson) Oudejans comb. \& stat. nov. Basionym: E. pubescens Vahl ['Desf.'] subvar. integrifolia J.-B.Deysson 1907, Actes Soc. Linn. Bordeaux 62: 33.

Euphorbia hirsuta Nathorst in Linnaeus f. laevis (Merino) Oudejans comb. \& stat. nov. Basionym: E. pubescens Vahl var. laevis B.Merino [y Román] 1906, Fl. Galicia 2: 532.

Euphorbia hirsuta Nathorst in Linnaeus f. serrata (Lojacono Pojero) Oudejans comb. \& stat. nov. Basionym: E. pubescens Vahl ['Desf.'] var. serrata M. Lojacono Pojero 1904-1907, Fl. sicul. 2(2): 333 .

Euphorbia hirsuta Nathorst in Linnaeus f. serrulatifolia (Deysson) Oudejans comb. \& stat. nov. Basionym: E. pubescens Vahl ['Desf.'] subvar. serrulatifolia J.-P. Deysson 1907, Actes Soc. Linn. Bordeaux 62: 33.

Euphorbia hirsuta Nathorst in Linnaeus f. subglabra (Godron in Grenier \& Godron) Oudejans comb. \& stat. nov. Basionym: E. pubescens Vahl var. subglabra [D. A.] Godron in [J. C. M.] Grenier \& [D. A.] Godron 1855, Fl. France 3: 79.

Euphorbia hyssopifolia Linnaeus var. blanchetii (Miquel ex Boissier in DC) Oudejans comb. nov. Basionym: E. brasiliensis Lamarck var. blanchetii ['Blancheti'] Miquel ex [P.] E. Boissier in A. P. de Candolle 1862, Prodr. 15(2): 24.

Euphorbia hyssopifolia Linnaeus var. paraguayensis (Chodat) Oudejans comb. nov. Basionym: E. brasiliensis Lamarck var. paraguayensis R. Chodat 1902 ['1901'], Bull. Herb. Boiss. (Ser. 2) $1: 399$.

Euphorbia hyssopifolia Linnaeus var. pruinosa (Chodat) Oudejans comb. \& stat. nov. Basionym: E. brasiliensis Lamarck f. pruinosa R. Chodat 1902 ['1901'], Bull. Herb. Boiss. (Ser. 2) 1: 399.

Euphorbia hyssopifolia Linnaeus var. pulchella (Kunth in HBK) Oudejans comb. \& stat. nov. Basionym: E. pulchella C. S. Kunth in Humboldt, Bonpland \& Kunth 1817, Nov. gen. sp. 2: 56.

Euphorbia hyssopifolia Linnaeus var. uniflora (Chodat \& Hassler) Oudejans comb. nov. Basionym: E. brasiliensis Lamarck var. uniflora R. Chodat \& E. Hassler 1905, Bull. Herb. Boiss. (Ser. 2) 5: 681.

Euphorbia inaequilatera Sonder var. jemenica (Schweinfurth) Oudejans comb. nov. Basionym: E. sanguinea Hochstetter \& Steudel ex Boissier in DC var. jemenica G. [A.] Schweinfurth 1899, Bull. Herb. Boiss. (Ser. 1) 7 (Appendix 2): 315.

Euphorbia insulana Vellozo in Arrabida var. tovarensis (Boissier) Oudejans comb. \& stat. nov.

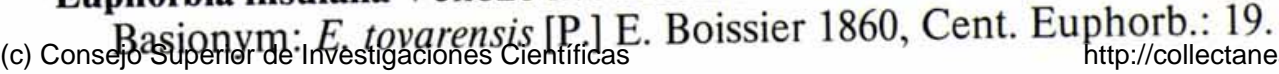


Euphorbia insulana Vellozo in Arrabida var. pilcomayensis (Croizat) Oudejans stat. nov. Basionym: E. insulana ssp. pilcomayensis L. [C. M.] Croizat 1943, J. Arnold Arbor. 24: 178.

Euphorbia jolkinii Boissier f. insularis (Hurusawa) Oudejans comb. nov. Basionym: Galarrhoeus ['Galarhoeus'] jolkinii (Boissier) Hara f. insularis I. Hurusawa 1954, J. Fac. Sci. Univ. Tokyo (Sect. 3 Bot.) 6(6): 254.

Euphorbia klotzschii Oudejans var. argentina (Muell. Arg. ex Grisebach) Oudejans comb. nov. Basionym: E. ovalifolia ([Engelmann ex] Klotzsch \& Garcke ex Klotzsch) Boissier in DC var. argentina J. Mueller Arg. ex A. [H. R.] Grisebach 1874, Abh. k. Ges. Wiss. Göttingen (Phys. Cl.) 19: 99.

Euphorbia klotzschii Oudejans var. schizosepala (Engelmann ex Boissier in DC) Oudejans comb. nov. Basionym: E. ovalifolia ([Engelmann ex] Klotzsch \& Garcke ex Klotzsch) Boissier in DC var. schizosepala Engelmann ex [P.] E. Boissier in A. P. de Candolle 1862, Prodr. 15(2): 43 .

Euphorbia lecheoides Millspaugh var. exumensis (Millspaugh) Oudejans comb. \& stat. nov. Basionym: Chamaesyce exumensis C. F. Millspaugh 1909, Field Columb. Mus. Bot. Ser. 2: 301-302.

Euphorbia lecheoides Millspaugh var. wilsonii (Millspaugh) Oudejans comb. \& stat. nov. Basionym: Chamaesyce wilsonii ['Wilsonii'] C. F. Millspaugh 1909, Field Columb. Mus. Bot. Ser. 2: 301.

Euphorbia marschalliana Boissier in DC ssp. armena (Prokhanov in Komarov) Oudejans comb. \& stat. nov. Basionym: E. armena Ya. I. Prokhanov in V. L. Komarov 1949, Fl. SSSR 14: 741-742.

Euphorbia mesembryanthemifolia Jacquin var. flexuosa (Kunth in HBK) Oudejans comb. \& stat. nov. Basionym: E. flexuosa C. S. Kunth in Humboldt, Bonpland \& Kunth 1817, Nov. gen. sp. 2: 55 .

Euphorbia mesembryanthemifolia Jacquin f. florida (Millspaugh) Oudejans comb. nov. Basionym: E. buxifolia Lamarck f. florida C. F. Millspaugh 1900, Field Columb. Mus. Bot. Ser. 2(1): 61 .

Euphorbia mesembryanthemifolia Jacquin var. ramosissima (Millspaugh) Oudejans comb. nov. Basionym: E. buxifolia Lamarck var. ramosissima C. F. Millspaugh 1900, Field Columb. Mus. Bot. Ser. 2(1): 61 .

Euphorbia mesembryanthemifolia Jacquin $\mathrm{f}$. reclinata (Millspaugh) Oudejans comb. nov. Basionym: E. buxifolia Lamarck f. reclinata C. F. Millspaugh 1900, Field Columb. Mus. Bot. Ser. 2(1): 60 .

Euphorbia mesembryanthemifolia Jacquin f. seminuda (Millspaugh) Oudejans comb. nov. Basionym: E. buxifolia Lamarck f. seminuda C. F. Millspaugh 1900, Field Columb. Mus. Bot. Ser. 2(1): 60.

Euphorbia nicaeensis Allioni ssp. volgenis (Kryshtofovich) Oudejans comb. \& stat. nov. Basionym: E. volgensis A. [N.] Kryshtofovich ['Krishtofovich'] 1929, Izv. Glavn. Bot. Sada SSSR 28: $375-378$.

Euphorbia pekinensis Ruprecht in Maximowicz f. denudata (Hurusawa) Oudejans comb. nov. Basionym: E. imaii Hurusawa f. denudata I Hurusawa 1940, J. Jap. Bot. 16: 577.

Euphorbia pekinensis Ruprecht in Maximowicz var. lasiocaula (Boissier in DC) Oudejans comb. \& stat. nov. Basionym: E. lasiocaula [P.] E. Boissier in A. P. de Candolle 1866, Prodr. 15(2): 1266. 
Euphorbia pekinensis Ruprecht in Maximowicz var. pseudolucorum (Hurusawa) Oudejans comb. nov. Basionym: Galarrhoeus ['Galarhoeus'] lasiocaulus (Boissier in DC) var. pseudolucorum ['pseudo-lucorum'] I. Hurusawa 1954, J. Fac. Sci. Univ. Tokyo (Sect. 3 Bot.) 6(6): 267.

Euphorbia pekinensis Ruprecht in Maximowicz var. sinanensis (Hurusawa) Oudejans stat. nov. Basionym: E. pekinensis f. sinanensis I. Hurusawa 1940, J. Jap. Bot. 16: 636.

Euphorbia pekinensis Ruprecht in Maximowicz var. watanabei (Makino) Oudejans comb. \& stat. nov. Basionym: E. watanabei ['Watanabei'] T. Makino 1920, J. Jap. Bot. 2(4): 14.

Euphorbia pithyusa Linnaeus var. dianthifolia (Lojacono Pojero) Oudejans comb. nov. Basionym: E. requienii ['Requieni'] Lojacono Pojero var. dianthifolia M. Lojacono Pojero 1917, Malpighia 28: 103-104.

Euphorbia rigida Marschall von Bieberstein var. mauretanica (Maire) Oudejans comb. nov. Basionym: E. biglandulosa Desfontaines var. mauretanica R. [C. J. E.] Maire 1929, Bull. Soc. Hist. Nat. Afr. Nord 20: 37.

Euphorbia rothiana Sprengel var. pubescens (Boissier in DC) Oudejans comb. nov. Basionym: E. oreophila Miquel var. pubescens [P.] E. Boissier in A. P. de Candolle 1862, Prodr. 15(2): 156.

Euphorbia rothiana Sprengel var. wightiana (Boissier) Oudejans comb. \& stat. nov. Basionym: E. wightiana ['Wightiana'] [P.] E. Boissier 1860, Cent. Euphorb.: 37.

Euphorbia salicifolia Host f. pseudosalicifolia (Schur) Oudejans stat. nov. Basionym: E. salicifolia var. pseudosalicifolia ['Pseudo-salicifolia'] [P. J.] F. Schur 1866, Enum. pl. Transsilv.: 597.

Euphorbia segetalis Linnaeus ssp. linifolia (Nathorst in Linnaeus) Oudejans comb. \& stat. nov. Basionym: E. linifolia [T. E. Nathorst in] C. Linnaeus 1759, Amoen. Acad. 4: 483 [=E. pinea Linnaeus 1767].

Euphorbia seguieriana Necker var. arenivaga (Martrin-Donos) Oudejans comb. nov. Basionym: E. gerardiana Jacquin var. arenivaga [J.] V. de Martrin-Donos 1864, Fl. Tarn: 624.

Euphorbia seguieriana Necker var. dentata (Chabert) Oudejans comb. nov. Basionym: $E$. gerardiana Jacquin var. dentata A. Chabert 1871, Bull. Soc. Bot. France 18: 199.

Euphorbia seguieriana Necker var. firma (Ledebour) Oudejans comb. \& stat. nov. Basionym: $E$. firma C. F. von Ledebour 1850, Fl. ross. 3(2): 563.

Euphorbia seguieriana Necker var. intermedia (Săvulescu) Oudejans comb. nov. Basionym: $E$. gerardiana Jacquin var. intermedia T. Săvulescu 1923 ['1922-1923'], Bull. Acad. Sci. Acad. Roum. 8: 115.

Euphorbia seguieriana Necker var. lanceolata (Cariot) Oudejans comb. nov. Basionym: $E$. gerardiana Jacquin var. lanceolata [A.] Cariot 1872, Étude fl. (ed. 5) 2: 539.

Euphorbia seguieriana Necker var. latifolia (Schmitz \& Regel) Oudejans comb. nov. Basionym: E. gerardiana Jarquin var. latifolia J. J. Schmitz \& Ed. Regel 1841, Fl. bonn.: 161.

Euphorbia seguieriana Necker var. sturii (Holuby) Oudejans comb. nov. Basionym: E. gerardiana Jacquin var. sturii ['Stúrii'] J. L. Holuby 1856, Verh. Ver. Naturk. Presburg 1: 18.

Euphorbia serrata Linnaeus f. ternata (Coutinho) Oudejans stat. nov. Basionym: E. serrata var. ternata A. X. P. Coutinho 1913, Fl. Portugal (ed. 1): 385.

Euphorbia serrata Linnaeus f. subacaulis (Rouy) Oudejans stat. nov. Basionym: E. serrata var. subacaulis G. [C. Ch.] Rouy 1910, Fl. France 12: 177. 
Euphorbia sieboldiana Morren \& Decaisne var. grandifolia (Franchet \& Savatier ex Hurusawa) Oudejans comb. \& stat. nov. Basionym: Galarrhoeus ['Galarhoeus'] sieboldianus (Morren \& Decaisne) Hara f. grandifolius Franchet \& Savatier ex I. Hurusawa 1954, J. Fac. Sci. Univ. Tokyo (Sect. 3 Bot.) 6(6): 243.

Euphorbia sieboldiana Morren \& Decaisne var. idzuensis (Hurusawa) Oudejans stat. nov. Basionym: E. sieboldiana f. idzuensis I. Hurusawa 1940, J. Jap. Bot. 16: 449.

Enphorbia sieboldiana Morren \& Decaisne var. sylvatica (Hurusawa) Oudejans stat. nov. Basionym: E. sieboldiana f. sylvatica Hurusawa 1940, J. Jap. Bot. 16: 449.

Euphorbia spathulata Lamarck var. mexicana (Engelmann in Emory) Oudejans comb. nov. Basionym: E. dictyosperma Fischer \& Meyer var. mexicana G. [T.] Engelmann in W. H. Emory 1858, Rep. U. S. Mex. bound. 2(1): 191.

Euphorbia squamosa Willdenow var. serrata (Boissier in DC) Oudejans comb. nov. Basionym: E. aspera Marschall von Biebertein var. serrata [P.] E. Boissier in A. P. de Candolle 1862, Prodr. 15(2): 124.

Euphorbia squamosa Willdenow var. talyschensis (Boissier \& Buhse) Oudejans comb. \& stat. nov. Basionym: E. talyschensis [P.] E. Boissier \& F. Buhse 1860, Nouv. Mém. Soc. Natural. Moscou 12: 196.

Euphorbia squamosa Willdenow var. wilhelmsiana (C. Koch) Oudejans comb. nov. Basionym: E. muricata Marschall von Bieberstein var. wilhelmsiana ['Wilhelmsiana'] C. Koch 1849 ['1848'], Linnaea 21: 725 .

Euphorbia stricta Linnaeus f. pubescens (Erdner) Oudejans stat. nov. Basionym: E. stricta var. pubescens E. Erdner 1911, Ber. naturwiss. Ver. Schwaben Neuburg 39-40: 569.

Euphorbia taurinensis Allioni var. brachyceras (Candargy) Oudejans comb. nov. Basionym: $E$. graeca Boissier \& Spruner ex Boissier var. brachyceras P. [-C.] Candargy 1898, Bull. Soc. Bot. France 45: 182.

Euphorbia taurinensis Allioni var. isophylla (K. Malý) Oudejans comb. \& stat. nov. Basionym: E. dalmatica Visiani f. isophylla K. Malý 1908, Glasn. Zemaljsk. Muz. Bosn. Hercegov. 20(4): 556.

Euphorbia togakusensis Hayata f. intermedia (Hurusawa) Oudejans comb. nov. Basionym: Galarrhoeus ['Galarhoeus'] togakusensis (Hayata) Hara f. intermedius I. Hurusawa 1954, J. Fac. Sci. Univ. Tokyo (Sect. 3 Bot.) 6(6): 269.

Euphorbia vachellii Hooker \& Arnott var. pubescens (Warnurg) Oudejans comb. nov. Basionym: E. serrulata Reinwardt var. pubescens O. Warburg 1891, Bot. Jahrb. 13: 347.

Euphorbia verrucosa Linnaeus f. montana (Gaudin) Oudejans stat. nov. Basionym: E. verrucosa var. montana J. F. A. [G. P.] Gaudin 1828, Fl. helv. 3: 284.

Euphorbia verrucosa Linnaeus f. velutina (Boissier in DC) Oudejans stat. nov. Basionym: $E$. verrucosa Linnaeus ['Lam.'] var. velutina [P.] E. Boissier in A. P. de Candolle 1862, Prodr. 15(2): 129.

Euphorbia verrucosa Linnaeus f. viridis (Erdner) Oudejans stat. nov. Basionym: E. verrucosa Linnaeus ['Lam.'] var. viridis E. Erdner 1911, Ber. naturwiss. Ver. Schwaben Neuburg 39-40: 569.

Euphorbia villosa Waldstein \& Kitaibel ex Willdenow ssp. semivillosa (Prokhanov) Oudejans comb. \& stat. nov. Basionym: Tithymalus semivillosus Ya. I. Prokhanov 1933, Consp. Syst. 
Euphorbia waldsteinii (Soják) A. Radcliffe-Smith ssp. saratoi (Ardoino) Oudejans comb. \& stat. nov. Basionym: E. saratoi ['Sarati’ sic] O. [= H. J. B.] Ardoino 1867, Fl. anal. Alpes-mar. (ed. 1): 335 .

\section{Literature cited}

CRoizat, L. (1940). New and critical Euphorbiaceae from eastern tropical Asia. J. Arnold Arbor. 21: 490-510.

FISCHER, F. [E. L. von] (1812). Descriptio plantarum rariorum Sibiriae. Bull. Soc. Natural. Moscou 3: (56)-(82).

FisCHER, F. [E. L. von] \& C. [A. von] MEYER (1835). Index seminum, quae Hortus botanicus imperialis petropolitanus pro mutua commutatione offert [I]. St. Petersburg.

OudEJANS, R. C. H. M. (1990). World catalogue of species names pubished in the tribe Euphorbieae (Euphorbiaceae) with their geographical distribution. Utrecht.

StEudEL, E. T. [= E. G. von] (1840-1841). Nomenclator botanicus. Editio secunda. Stuttgardiae et Tubingae (J. G. Colla).

TurCZANINOW, N. (1838). Catalogus plantarum in regionibus baicalensibus, et in Dahuria sponte crescentium. Bull. Soc. Imp. Natural. Moscou 9: 85-107.

(Received and accepted 14 February 1992) 